\title{
Experiencia del Hospital de Especialidades del Centro Médico Nacional La Raza en pacientes con trauma vascular atendidos inicialmente en otra unidad
}

\section{Tertiary care hospital of the National Medical Center La Raza experience in patients with vascular trauma initially treated in another unit}

\author{
Sofia L. Muñoz-Vega, Héctor Bizueto-Rosas*, Alfonso Cossío-Zazueta, Roberto C. Serrato-Auld y \\ Melanie Segoviano-Sandoval \\ Departamento de Angiología, Hospital de Especialidades, Centro Médico La Raza, Instituto Mexicano del Seguro Social, Ciudad de México, México
}

\begin{abstract}
Resumen
Antecedentes: Las lesiones vasculares principales causas de morbimortalidad en jóvenes. El retraso de la cirugía o no contar con un cirujano vascular son condicionantes de complicaciones, como el síndrome compartimental, necrosis muscular, amputación o la muerte. Nuestro objetivo fue describir la frecuencia y complicaciones de los pacientes con trauma vascular atendidos en otros hospitales y referidos al tercer nivel. Método: Se realizó un estudio retrospectivo, de marzo de 2014 a marzo de 2019, en los expedientes de derechohabientes del Hospital de Especialidades con antecedentes de trauma vascular de las extremidades: evolución, quién los atendido. Resultados: Fueron 88 pacientes, el $88 \%$ de sexo masculino, el 43\% mayores de 30 años de edad, el 13\% con comorbilidades, el 96\% atendidos en otra unidad, el 38\% con lesiones por arma de fuego, el 79\% un solo vaso, el 70\% con una escala de Mangled Extremity Severity Score < 7, el 26\% con lesión en la arteria femoral y el 65\% retraso en el traslado mayor a 12 h. Complicaciones ( $p=0.004)$ : dermofasciotomía (27\%), amputación (25\%). Mortalidad (1.0\%). Conclusiones: El tiempo de evolución y la atención primaria inciden en la evolución de estos pacientes. La atención por otro cirujano no vascular es aceptable pero no idónea.
\end{abstract}

Palabras clave: Trauma vascular. Cirujano vascular. Complicaciones.

\section{Abstract}

Background: Vascular injuries are the main cause of morbidity and mortality among young patients. Delay of surgery and not having a vascular surgeon is a conditioning factor of several complications such as muscular damage, necrosis, mayor limb amputation or even death. Our main objective was to describe the frequency and complications of patients with vascular trauma first attended in other center and then transferred to a reference center. Method: A retrospective, observational study was performed with records of patients admitted for vascular limb trauma from March 2014 to March 2019 analyzing trauma, evolution and first attending center. Results: Eighty-eight patients, $88 \%$ male, 43\% older than 30 years old, comorbi-

Correspondencia:

*Héctor Bizueto-Rosas

E-mail: dr_bizueto_h@yahoo.com
Disponible en internet: 20-09-2019

Rev Mex Angiol. 2019;47(3):22-29 www.RMAngiologia.com

0377-4740/@ 2019 Sociedad Mexicana de Angiología y Cirugía Vascular y Endovascular, A.C. Published by Permanyer México. This is an open access article under the CC BY-NC-ND license (http://creativecommons.org/licenses/by-nc-nd/4.0/). 
dities in $13 \%, 96 \%$ of them were previously admitted in other centers, $38 \%$ with gun injury, single vessel injury in $79 \%$, mangled extremity severity score scale $<7$ in $70 \%$, femoral artery injury in $26 \%$, delay of transfer greater than $12 \mathrm{~h}$ in $65 \%$, complications $p=0.004$, fasciotomy required in $27 \%$, mayor limb amputation $25 \%$, mortality $1.0 \%$. Conclusions: Primary care, time of evolution and initial management have an impact on rate of complications and prognosis. Initial management by a non-vascular surgeon is acceptable but not ideal.

Key words: Vascular trauma. Vascular surgeon. Complications.

\section{Introducción}

Siendo el trauma vascular una de las primeras causas de muerte en adultos jóvenes en países industrializados y causa importante de pérdida de años de vida productiva, resulta indispensable conocer los factores determinantes para reducir las complicaciones y la pérdida de extremidades. En México, el porcentaje de amputación mayor secundario a trauma vascular es cercano al $25 \%$.

En EE.UU., en el 2010, la mortalidad por trauma de 1 a 24 años de edad fue del $63 \%$, y de 25 a 44 , del $42 \%$. Aproximadamente 5 millones de personas mueren anualmente como resultado de trauma en este grupo etario.

La incidencia de lesión vascular es de 1.6\% en adultos y $0.6 \%$ para pacientes pediátricos. En México, en el 2011, entre las principales causas de mortalidad general en la población pediátrica (5-19 años de edad), hubo los accidentes: el $30 \%$ de estos pacientes tienen algún tipo de lesión vascular'.

Del 60 al $90 \%$ de las lesiones civiles en el tronco o las extremidades son secundarias a mecanismos penetrantes, generalmente por armas de fuego. Las lesiones por trauma cerrado son infrecuentes, por lo general en pacientes de mayor edad; al implicar mecanismos de mayor fuerza y velocidad, se asocian con lesiones intrabdominales, torácicas, de tejidos blandos u óseos ${ }^{2-5}$.

Las lesiones vasculares mayores son responsables del $20 \%$ de la mortalidad ${ }^{2}$. Son raras en el campo civil: el desarrollo de maquinaria y vehículos automotores, el incremento de la violencia y la disposición de armas han aumentado la incidencia. La incidencia exacta y distribución de los mecanismos de lesión varían entre diferentes centros, dependiendo de la población y el escenario (urbano versus rural) ${ }^{2,6}$. La incidencia de trauma vascular mayor tiende a subestimarse, pues no incluye los pacientes que mueren en la escena de trauma $o$ al llegar al hospital. La mayoría de las muertes prehospitalarias o inmediatas por lesión vascular fueron por lesión aórtica (55\%); el 78\% estuvieron asociadas con muerte en los primeros 15 minutos $^{2,6-8}$.
En el Hospital General de México, en 2017, se encontró que las causas más frecuentes fueron por proyectil de arma de fuego (42\%), objetos punzocortantes (16\%), contusión secundaria a accidentes automovilísticos (19\%) y trauma iatrogénico $(24 \%)$. Las locaciones más frecuentes fueron las extremidades inferiores (52\%), las extremidades superiores $(29 \%)$ y la arteria femoral superficial, seguida de la arteria braquial y la subclavia fueron las más afectadas $^{9-11}$.

Las lesiones en miembros inferiores se asocian generalmente a accidentes en motocicleta y automóvil; entre otras causas se incluye el trauma iatrogéni$\mathrm{co}^{7,12,13}$. Las lesiones de miembros superiores se deben generalmente a heridas penetrantes ${ }^{14-16}$.

La lesión vascular mayor y el sangrado son responsables del $25 \%$ de las muertes tempranas. La edad promedio de presentación en los pacientes con lesión vascular aumentó 10 años entre 1996 y 2004. Ahora tenemos pacientes de mayor edad con enfermedad vascular preexistente, lo que puede incrementar el riesgo por comorbilidades y alterar las opciones de tratamiento ${ }^{17}$.

En los países desarrollados, con menor tiempo de traslado y el avance en los cuidados prehospitalarios, la mortalidad prehospitalaria es del 0.5 al 1.5\%. En pacientes gravemente lesionados, las técnicas de control de daños (cirugía abreviada, resucitación y shunts intravasculares temporales) han disminuido la mortalidad y aumentado la tasa de salvamento de extremidades ${ }^{14}$. Los shunts, al restituir el flujo, mantienen viable la extremidad y proporcionan un control relativo de la hemorragia, hasta la revascularización en un centro especializado $0^{9,10,12,13}$.

Un factor determinante para decidir si se realiza reparación y salvamento de la extremidad es el tiempo que transcurre entre la lesión y el tratamiento. En México, el porcentaje de amputación mayor es del $25 \%$, por retraso en el transporte del paciente, diagnósticos erróneos, carencia de un cirujano capacitado en trauma vascular que conozca las implicaciones que tiene el síndrome de reperfusión y la urgencia de la 
Tabla 1. Escala mangled extremity severity score (MESS)

\begin{tabular}{|l|l|}
\hline Escala mess & \\
\hline Lesión ósea y tejidos blandos & 1 \\
\hline Baja energía (herida cortante, fractura simple) & 2 \\
\hline Energía media (fractura conminuta, herida contusa) & 3 \\
\hline $\begin{array}{l}\text { Alta energía (aplastamiento, arma de fuego militar) } \\
\text { Muy alta energía (con gran avulsión de tejidos blandos) }\end{array}$ & 4 \\
\hline Isquemia de la extremidad* & \\
\hline $\begin{array}{l}\text { Pulso reducido, con perfusión normal } \\
\text { Sin pulso, parestesias con llenado capilar retardado }\end{array}$ & 1 \\
\hline Frialdad, parálisis, anestesia & 2 \\
\hline Estado de choque & 3 \\
\hline $\begin{array}{l}\text { Presión arterial mayor de } 90 \text { mmhg } \\
\text { Hipotensión transitoria }\end{array}$ & \\
\hline Hipotensión persistente & 0 \\
\hline Edad (años) & 1 \\
\hline Menor de 30 & 2 \\
\hline $30-50$ & 0 \\
\hline Mayor de 50 & 1 \\
\hline
\end{tabular}

* Si el tiempo de isquemia es mayor a 6 horas se duplica. MESS: Mangled Extremity Severity Score.

reparación vascular temprana (antes de 6 horas) $)^{10,16}$. Las complicaciones se deben principalmente al retraso o desaciertos en el diagnóstico y tratamiento; pueden presentarse a corto o largo plazo ${ }^{14}$. En Brasil observaron que los pacientes que recorrieron distancias largas antes de recibir atención especializada presentaron periodos de hospitalización prolongados, mayores complicaciones, como amputación de extremidades. La distancia estuvo estadísticamente relacionada con una mayor probabilidad de amputación de las extremidades ${ }^{15}$ : «la realización de una amputación en trauma vascular es directamente proporcional a la distancia del lugar del accidente al centro de trauma.»

En EE.UU., del 2008 al 2013, se realizó un estudio en pacientes que requirieron reparación quirúrgica por lesiones vasculares traumáticas, comparando los resultados de pacientes tratados por cirujanos vasculares versus cirujanos de trauma. Los pacientes atendidos por cirujanos vasculares tuvieron lesiones más severas de acuerdo a la escala de MESS (Mangled Extremity Severity Score) (Tabla 1). Demostraron que la reparación vascular por cirujanos de trauma es segura, pero los cirujanos vasculares continúan ejerciendo un papel significativo en el tratamiento quirúrgico, sobre todo en lesiones vasculares graves, con un puntaje alto ${ }^{9}$.

\section{Métodos}

Se realizó un estudio retrospectivo, incluyendo los pacientes atendidos en el Hospital de Especialidades del Centro Médico Nacional La Raza por traumatismo vascular que inicialmente fueron atendidos en otra unidad médica y trasladados a este nosocomio, de marzo de 2014 a marzo de 2019, y se identificaron las comorbilidades y evolución en el postoperatorio inmediato y tardío. Dicho protocolo fue aprobado previamente por el comité de investigación y de ética de nuestro hospital, y por tratarse de un estudio retrospectivo, no requirió consentimiento informado.

La información se obtuvo de los expedientes clínicos, desde su ingreso a nuestro servicio hasta el periodo determinado. El objetivo del estudio fue evaluar si estos pacientes con trauma vascular de las extremidades tienen mayor frecuencia de complicaciones y mayor tasa de amputación en relación con los atendidos inicialmente en nuestra unidad.

Se realizó un análisis estadístico de regresión múltiple logística binaria con el fin de establecer la influencia de las variables independientes sobre la frecuencia, complicaciones y mortalidad. Se comparó los pacientes intervenidos en otra unidad con la nuestra.

Variable cualitativa ordinal, de acuerdo con el mecanismo, grado de lesión conforme la escala de MESS, tiempo de evolución, quién lo atendió.

\section{Resultados}

Fueron 88 pacientes, el $88 \%$ hombres, el $40.9 \%$ entre 30 y 49 años de edad, y el $43 \%$ menores de 30 años. El $96 \%$ se atendieron inicialmente en otra unidad médica, de estos, el $44 \%$ en un hospital de trauma del seguro social y el $45 \%$ en su hospital general de zona (Fig. 1). Las comorbilidades fueron: diabetes mellitus (40\%), hipertensión arterial sistémica (27\%), enfermedad renal crónica (13\%), epilepsia (13\%), cardiopatía (7\%) (Fig. 2).

Las extremidades más afectadas fueron las inferiores (59\%). Las lesiones por arma de fuego fueron el $37.5 \%$, le siguieron las lesiones contusas (17.0\%). De acuerdo con la escala de MESS, la mayoría con calificación menor a 7 (70\%). Presentaron comorbilidades el $13 \%$ de los pacientes (Fig. 2).

La arteria femoral fue el vaso más afectado $(26 \%)$, poplítea (16\%), humeral (12\%), vena femoral (9\%). En el $34 \%$ de los pacientes se interpuso un injerto (Fig. 3).

Las complicaciones se presentaron en el $77 \%$ de los pacientes; una complicación en el 62\% (Fig. 4).

Salvamento de la extremidad (74\%), mortalidad (1\%). En negritas la variable con significancia estadística $p=$ 0.004 (número de complicaciones en relación a tiempo de isquemia para número de complicaciones) (Tabla 3). 


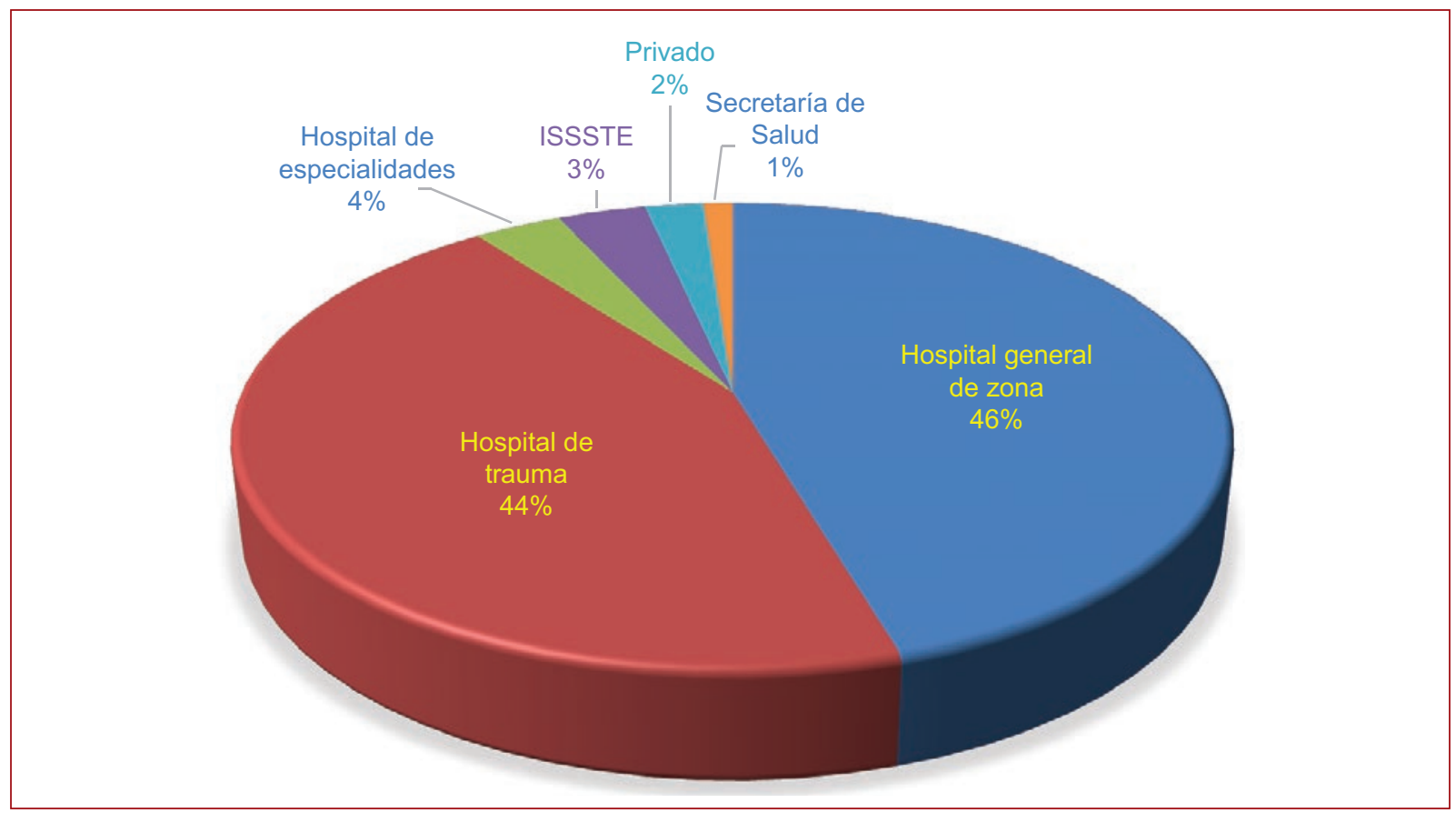

Figura 1. Hospitales de atención inicial.

ISSSTE: Instituto de Seguridad y Servicios Sociales de los Trabajadores del Estado.

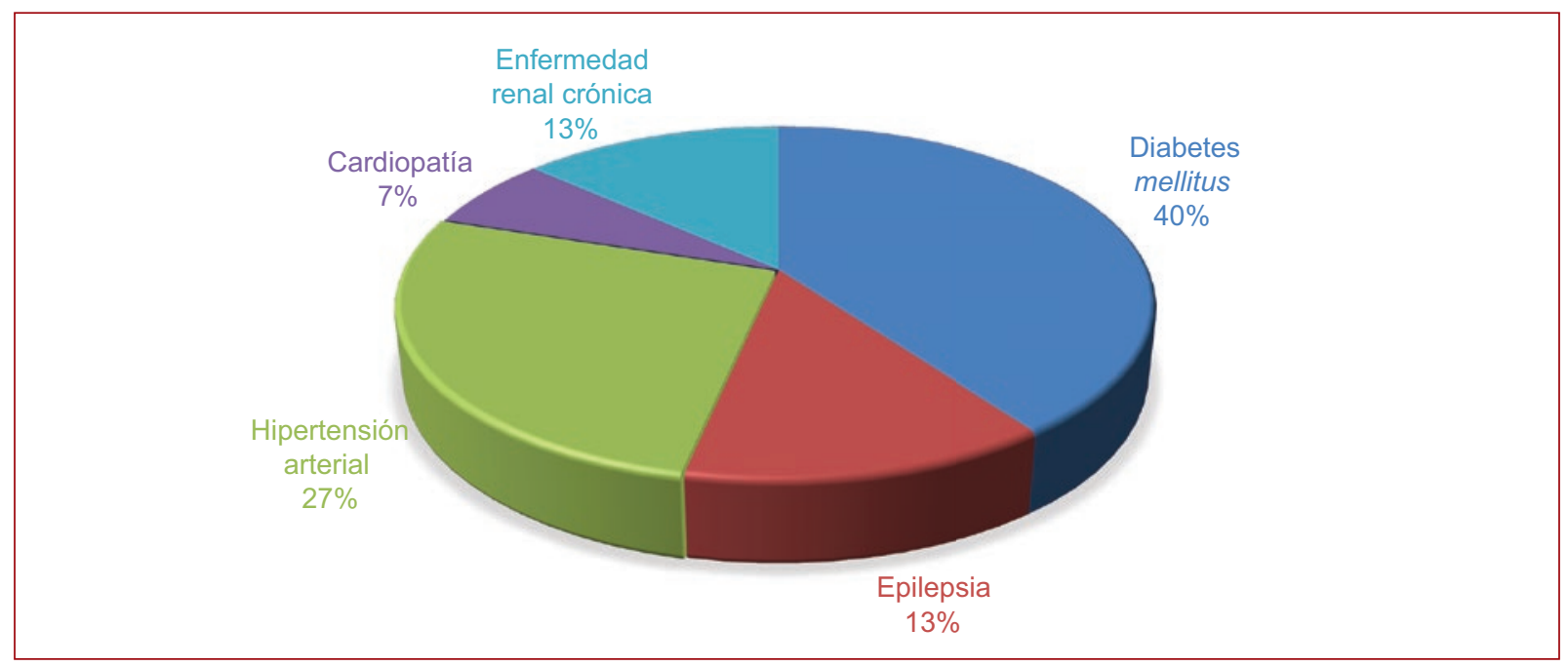

Figura 2. Comorbilidades identificadas. Comorbilidades identificadas.

\section{Discusión}

- Disponemos de 6 horas para restablecer la perfusión de los miembros inferiores.

- Si en las primeras 24 horas de postoperatorio falla una reconstrucción arterial o venosa, «es por falla técnica».

- El trauma vascular se traduce en sangrado excesivo o en la ausencia de este (anónimo)².
Con estas primicias, deberíamos siempre considerar la atención adecuada y oportuna de un trauma vascular.

La reparación de las lesiones vasculares deberá efectuarse con el paciente hemodinámicamente estable, con estabilidad esquelética, viabilidad tisular, condiciones adecuadas del lecho quirúrgico y con los recursos para practicar la cirugía. 


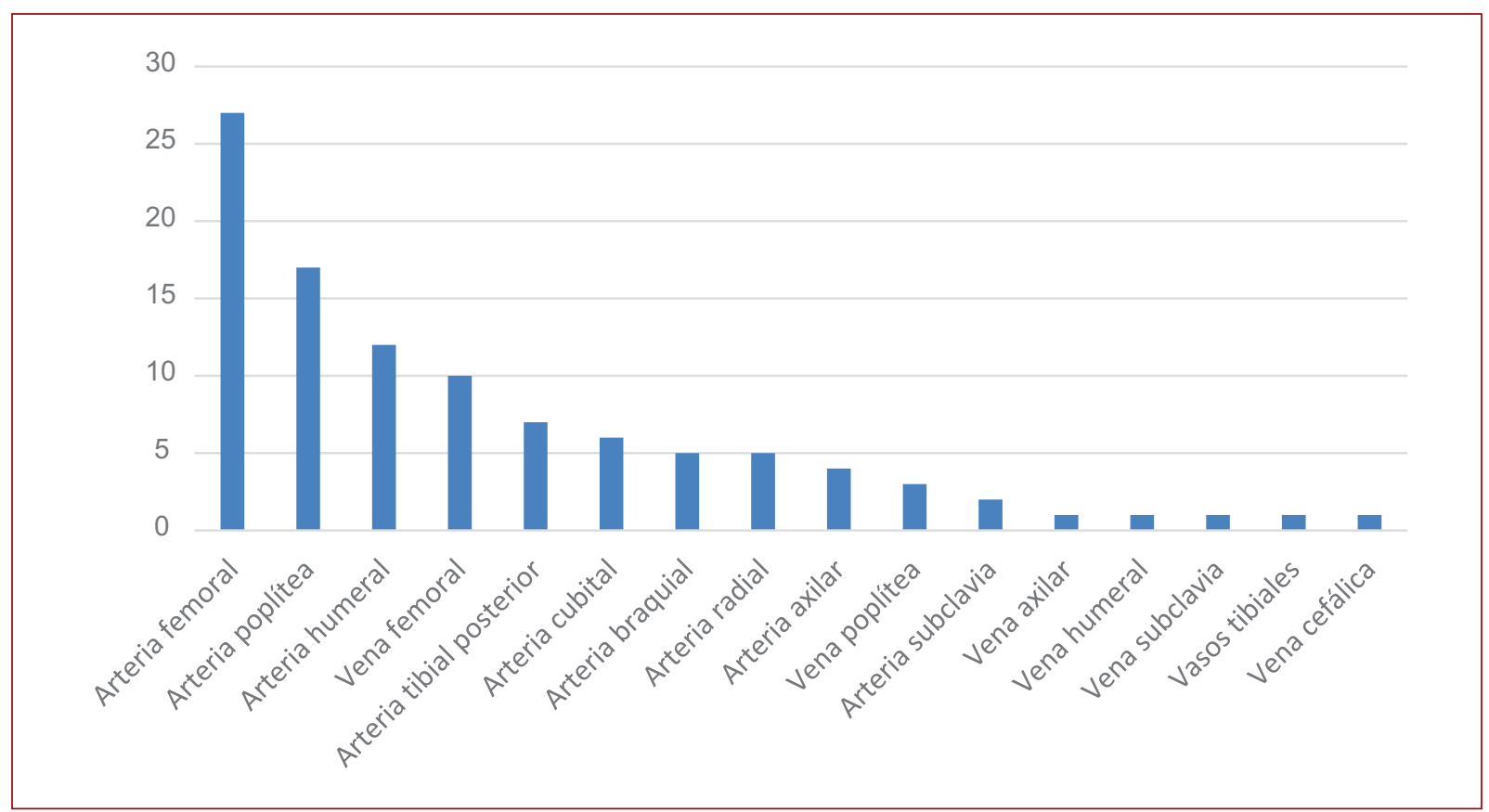

Figura 3. Afección de los vasos.

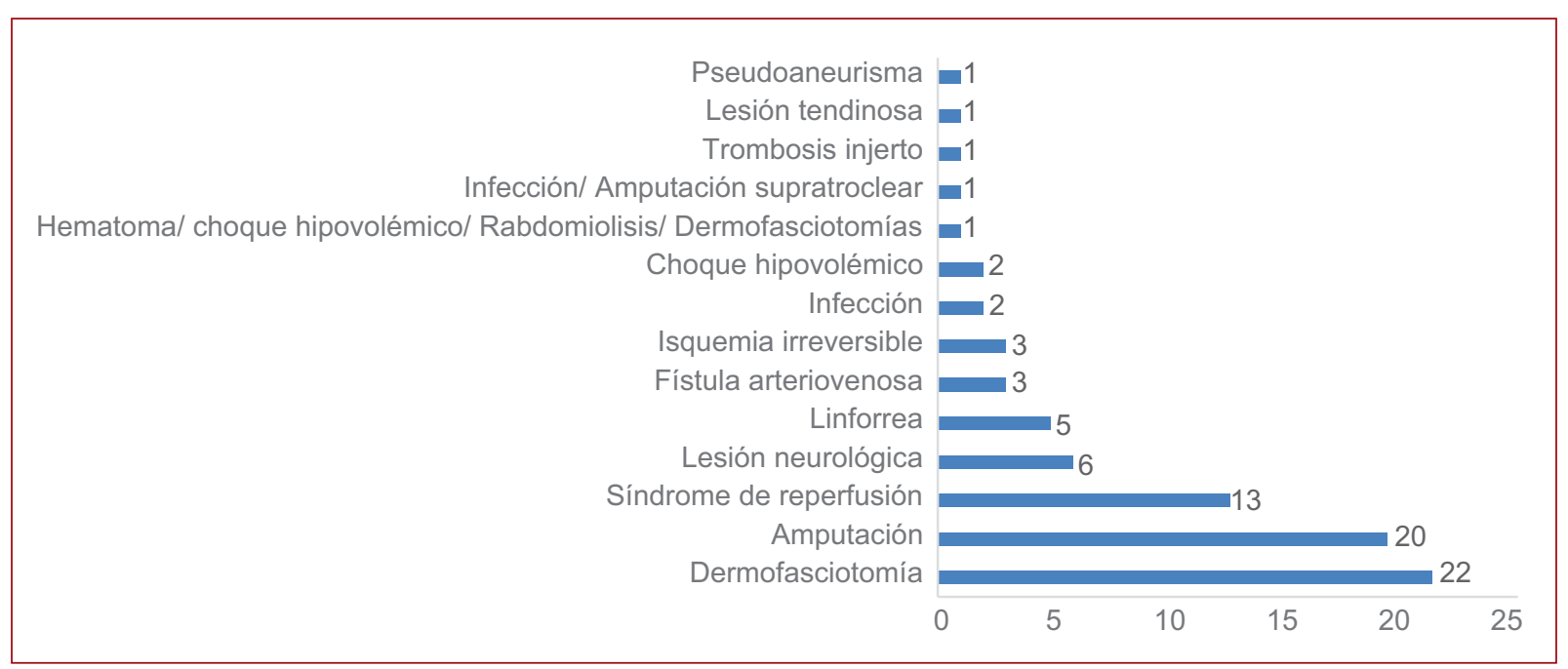

Figura 4. Complicaciones más fuertes.

El control de daños en el paciente con trauma vascular antes de ser trasladado a un servicio de trauma especializado es prioritario.

La cirugía vascular de emergencia en ocasiones es realizada por un cirujano general, que tiene el conocimiento pleno de la patología, las habilidades clínicas y técnicas en trauma vascular y conoce las implicaciones legales involucradas versus trasladar al enfermo a otro hospital de mayor especialización, todo ello condiciona que los resultados no siempre sean los óptimos ${ }^{16}$.
La mortalidad por una amputación secundaria a trauma sigue siendo alta, sobre todo cuando se acompaña de lesión venosa (25-30\%).

La carga global y el impacto del trauma como un agente de la muerte y la discapacidad está cada vez más bien caracterizado ${ }^{17,18}$

En cualquier hospital de trauma u hospital general, los pacientes de trauma son evaluados de acuerdo al protocolo de Apoyo vital avanzado en trauma: la evaluación de las extremidades, sobre todo las inferiores, 
Tabla 2. Características clínicas de la muestra de pacientes

\begin{tabular}{|c|c|c|c|c|c|}
\hline Variable & No. & $\%$ & Variable & No. & $\%$ \\
\hline \multicolumn{6}{|c|}{ Características clínicas } \\
\hline $\begin{array}{l}\text { Grupo de edad } \\
\quad<30 \\
30 \text { a } 49 \\
500+\end{array}$ & $\begin{array}{l}38 \\
36 \\
14\end{array}$ & $\begin{array}{l}43.2 \\
40.9 \\
15.9\end{array}$ & $\begin{array}{l}\text { Miembro afectado } \\
\text { Pélvico } \\
\text { Torácico }\end{array}$ & $\begin{array}{l}52 \\
36\end{array}$ & $\begin{array}{l}59.1 \\
40.9\end{array}$ \\
\hline $\begin{array}{l}\text { Sexo } \\
\text { Femenino } \\
\text { Masculino }\end{array}$ & $\begin{array}{l}11 \\
77\end{array}$ & $\begin{array}{l}12.5 \\
87.5\end{array}$ & $\begin{array}{l}\text { Hospital de atención inicial } \\
\text { Hospital de especialidades } \\
\text { Otro }\end{array}$ & $\begin{array}{c}3 \\
85\end{array}$ & $\begin{array}{c}3.4 \\
96.6\end{array}$ \\
\hline $\begin{array}{l}\text { Comorbilidades } \\
\text { Sí } \\
\text { No }\end{array}$ & $\begin{array}{l}11 \\
77\end{array}$ & $\begin{array}{l}12.5 \\
87.5\end{array}$ & $\begin{array}{l}\text { Intervención previa } \\
\text { No } \\
\text { Sí }\end{array}$ & $\begin{array}{l}64 \\
24\end{array}$ & $\begin{array}{l}72.7 \\
27.3\end{array}$ \\
\hline $\begin{array}{l}\text { Fractura asociada } \\
\text { No } \\
\text { Sí }\end{array}$ & $\begin{array}{l}66 \\
22\end{array}$ & $\begin{array}{l}75.0 \\
25.0\end{array}$ & $\begin{array}{l}\text { Tiempo de isquemia } \\
\quad<12 \text { horas } \\
120+\text { horas }\end{array}$ & $\begin{array}{l}33 \\
53\end{array}$ & $\begin{array}{l}37.5 \\
60.2\end{array}$ \\
\hline $\begin{array}{l}\text { Tipo de lesión } \\
\text { Aplastamiento } \\
\text { Arma de fuego } \\
\text { Contusa } \\
\text { Fractura } \\
\text { latrogénica } \\
\text { Punzocortante }\end{array}$ & $\begin{array}{c}9 \\
33 \\
15 \\
1 \\
7 \\
22\end{array}$ & $\begin{array}{c}10.2 \\
37.5 \\
17.0 \\
1.1 \\
8.0 \\
25.0\end{array}$ & $\begin{array}{l}\text { MESS } \\
\quad<7 \\
70+\end{array}$ & $\begin{array}{l}62 \\
26\end{array}$ & $\begin{array}{l}70.5 \\
29.5\end{array}$ \\
\hline \multicolumn{6}{|c|}{ Resultado final } \\
\hline $\begin{array}{l}\text { Complicaciones } \\
\text { No } \\
\text { Sí }\end{array}$ & $\begin{array}{l}20 \\
68\end{array}$ & $\begin{array}{l}22.7 \\
77.3\end{array}$ & $\begin{array}{l}\text { Salvamento de miembro } \\
\text { No } \\
\text { Sí }\end{array}$ & $\begin{array}{l}23 \\
65\end{array}$ & $\begin{array}{l}26.1 \\
73.9\end{array}$ \\
\hline $\begin{array}{l}\text { Número de complicaciones } \\
1 \\
20+\end{array}$ & $\begin{array}{l}42 \\
25\end{array}$ & $\begin{array}{l}61.8 \\
36.8\end{array}$ & $\begin{array}{l}\text { Mortalidad } \\
\text { No } \\
\text { Sí }\end{array}$ & $\begin{array}{c}87 \\
1\end{array}$ & $\begin{array}{r}98.9 \\
1.1\end{array}$ \\
\hline
\end{tabular}

pasa a un segundo término, solo que la lesión sea una hemorragia importante potencialmente mortal ${ }^{19}$. El pronóstico del paciente está ligado a diversos factores, y el más importante es el tiempo de isquemia. El tiempo de oro son 6 horas, no así para el tejido nervioso, que en 3 horas se daña y ocasiona déficits neurológicos motores o sensitivos ${ }^{20,21}$, y si recordamos como lo habíamos mencionado, la población joven (sector productivo) del sexo masculino es la más afectada: al transitar por las calles, está más expuesta a sufrir lesiones por armas de fuego u objetos punzocortantes, que afectan más a las extremidades inferiores $^{19}$, por lo que en un país como el nuestro, en el que el $28 \%$ de la población son hombres entre los 15 y los 45 años de edad, representa una carga económica importante.

Según la Organización Mundial de la Salud, las muertes por accidentes de tránsito registran una tasa de mortalidad de 20.1/100,000 habitantes por año en los países con ingresos económicos intermedios, como el nuestro. En México, durante el periodo de 2003 a 2007 ocurrieron en promedio 53,480 muertes anuales por lesiones, considerando todos los grupos de edad; de ellas, el $72 \%$ fueron accidentales. En cuanto al mecanismo, en 2011, la tasa de mortalidad por siniestros de tránsito fue de 14.4/100 mil habitantes, luego entonces, «El tratamiento vascular exitoso se basa en el diagnóstico y tratamiento rápidos» ${ }^{1,19}$.

Actualmente los traumas representan la tercera causa de muerte y la primera en menores de 45 años a nivel mundial. Los daños vasculares representan el $3 \%$ de los traumas en la población civil y se asocian a una gran morbimortalidad en pleno siglo XXI.

En países desarrollados, la tasa de rescate de la extremidad es cercana al $100 \%$, gracias al rápido traslado al hospital adecuado, atención temprana de los pacientes, diagnóstico precoz y tratamiento inmediato ${ }^{20}$.

Las lesiones vasculares producen daños que comprometen no solo la función, sino la vida del paciente. Las complicaciones relacionadas con esta patología 
Tabla 3. Significancia de Chi cuadrada para variables de resultado en relación con intervención previa

\begin{tabular}{|c|c|c|c|c|c|c|c|}
\hline \multirow[t]{2}{*}{ Intervención previa } & \multirow[t]{2}{*}{$\mathbf{n}$} & \multirow[t]{2}{*}{$\%$} & \multirow[t]{2}{*}{$\mathbf{n}$} & \multirow[t]{2}{*}{$\%$} & \multirow[t]{2}{*}{ Total } & \multicolumn{2}{|c|}{ Significancia exacta } \\
\hline & & & & & & Bilateral & Unilatera \\
\hline Tiempo de isquemia & \multicolumn{2}{|c|}{$<12 \mathrm{~h}$} & \multicolumn{2}{|c|}{$120+$} & & & \\
\hline $\begin{array}{l}\text { No } \\
\text { Sí } \\
\text { Total }\end{array}$ & $\begin{array}{c}25 \\
8 \\
33\end{array}$ & $\begin{array}{l}40 \\
35 \\
38\end{array}$ & $\begin{array}{l}38 \\
15 \\
53\end{array}$ & $\begin{array}{l}60 \\
65 \\
62\end{array}$ & $\begin{array}{l}63 \\
23 \\
86\end{array}$ & 0.804 & 0.439 \\
\hline Clasificación MESS & \multicolumn{2}{|c|}{$<7$} & \multicolumn{2}{|c|}{$70+$} & & & \\
\hline $\begin{array}{l}\text { No } \\
\text { Sí } \\
\text { Total }\end{array}$ & $\begin{array}{l}42 \\
20 \\
62\end{array}$ & $\begin{array}{l}66 \\
83 \\
70\end{array}$ & $\begin{array}{c}22 \\
4 \\
26\end{array}$ & $\begin{array}{l}34 \\
17 \\
30\end{array}$ & $\begin{array}{l}64 \\
24 \\
88\end{array}$ & 0.123 & 0.084 \\
\hline Complicaciones & \multicolumn{2}{|c|}{ No } & \multicolumn{2}{|c|}{ Sí } & & & \\
\hline $\begin{array}{l}\text { No } \\
\text { Sí } \\
\text { Total }\end{array}$ & $\begin{array}{c}15 \\
5 \\
20\end{array}$ & $\begin{array}{l}23 \\
21 \\
23\end{array}$ & $\begin{array}{l}49 \\
19 \\
68\end{array}$ & $\begin{array}{l}77 \\
79 \\
77\end{array}$ & $\begin{array}{l}64 \\
24 \\
88\end{array}$ & 1 & 0.52 \\
\hline Salvamento de la extremidad & \multicolumn{2}{|c|}{ No } & \multicolumn{2}{|c|}{ Sí } & & & \\
\hline $\begin{array}{l}\text { No } \\
\text { Sí } \\
\text { Total }\end{array}$ & $\begin{array}{c}16 \\
7 \\
23\end{array}$ & $\begin{array}{l}25 \\
29 \\
26\end{array}$ & $\begin{array}{l}48 \\
17 \\
65\end{array}$ & $\begin{array}{l}75 \\
71 \\
74\end{array}$ & $\begin{array}{l}64 \\
24 \\
88\end{array}$ & 0.787 & 0.443 \\
\hline Número de complicaciones & \multicolumn{2}{|c|}{1} & \multicolumn{2}{|c|}{$20+$} & & & \\
\hline $\begin{array}{l}\text { No } \\
\text { Sí } \\
\text { Total }\end{array}$ & $\begin{array}{c}36 \\
6 \\
42\end{array}$ & $\begin{array}{l}73 \\
33 \\
63\end{array}$ & $\begin{array}{l}13 \\
12 \\
25\end{array}$ & $\begin{array}{l}27 \\
67 \\
37\end{array}$ & $\begin{array}{l}49 \\
18 \\
67\end{array}$ & 0.004 & 0.003 \\
\hline Defunción & \multicolumn{2}{|c|}{ No } & \multicolumn{2}{|c|}{$\mathrm{Si}$} & & & \\
\hline $\begin{array}{l}\text { No } \\
\text { Sí } \\
\text { Total }\end{array}$ & $\begin{array}{l}64 \\
23 \\
87\end{array}$ & $\begin{array}{c}100 \\
96 \\
99\end{array}$ & $\begin{array}{l}0 \\
1 \\
1\end{array}$ & $\begin{array}{c}0 \\
4.2 \\
1.1\end{array}$ & $\begin{array}{l}64 \\
24 \\
88\end{array}$ & 0.273 & 0.273 \\
\hline
\end{tabular}

varían desde isquemia, sangrado a muerte, y cuando existe retraso en la atención, daño por reperfusión (rabdomiólisis, insuficiencia renal, falla multiorgánica e incluso la muerte), pueden presentarse complicaciones a corto o largo plazo aún con tratamientos quirúrgicos adecuados ${ }^{14}$.

Mattox, et al. documentaron que aumentó el 400\% el trauma cardiovascular en la población civil en Houston entre 1958-1988; el 50\% de ellas ocurridas en los últimos 10 años. Estas lesiones, como ocurre en países en vías de desarrollo como el nuestro, incluyen lesiones iatrogénicas (cateterismos, angiografías y procedimientos quirúrgicos, y la arteria femoral y braquial son las más lesionadas) 22,23

En nuestra investigación se presentaron situaciones que ameritaron su resolución por un cirujano especializado, como la colocación de un stent en dos pacientes (2.3\%) y la resección de un seudoaneurisma, lo que demuestra que lo ideal es que se cuente con angiología.
Si bien es cierto que «una de las principales fascinaciones en cirugía es el manejo de los vasos lesionados" (Halsted, 1912), cuando existe falla técnica y se requiere reoperación, la incidencia de infección aumenta considerablemente y «una infección en cirugía vascular es una pesadilla para el cirujano y el paciente».

«La mayor sabiduría que existe es conocerse a uno mismo." (Galileo Galilei).

Somos un país en desarrollo, con infraestructura hospitalaria deficiente, sobrepoblación de las principales ciudades, vehículos cada vez más rápidos, con el nivel de estrés y de agresividad en aumento por las condiciones económicas de nuestra población, por lo que se podría considerar al trauma vascular actualmente, como un problema de salud pública.

Tenemos que incidir como especialistas en la toma de decisiones con respecto a la salud y la organización de las unidades médicas, por lo menos en nuestro instituto, pues, actualmente, la población joven es la que 
predomina y es la que transita, y es la más expuesta a dichas lesiones.

\section{Conclusiones}

La estrategia terapéutica se basa en el tratamiento quirúrgico precoz y óptimo, ya que conlleva la mejor tasa de resultados satisfactorios en el trauma vascular. Hay una equivalencia respecto a los factores demográficos, mecanismos de la lesión, estrategias terapéuticas quirúrgicas, y resultados inmediatos satisfactorios entre lesiones arteriales penetrantes y contusas. El porcentaje de salvamento de extremidades fue aceptable con la intervención de un cirujano de trauma, pero no óptimo. La angiografía como modalidad diagnóstica y el tratamiento quirúrgico rápido son los principales factores que contribuyen al salvamento de la extremidad y prevención de las complicaciones. El abordaje multidisciplinario con un tratamiento oportuno conlleva resultados satisfactorios de las lesiones vasculares.

\section{Conflicto de intereses}

Los autores declaran que no existe conflicto de intereses.

\section{Agradecimientos}

Al MC. Dr. Javier Valdés Hernández, por su apoyo técnico con los cálculos estadísticos, gráficas y tablas. Al Dr. Hugo Alonso Pérez González, por su apoyo técnico en la revisión y traducción.

\section{Responsabilidades éticas}

Protección de personas y animales. Los autores declaran que para esta investigación no se han realizado experimentos en seres humanos ni en animales.

Confidencialidad de los datos. Los autores declaran que han seguido los protocolos de su centro de trabajo sobre la publicación de datos de pacientes.

Derecho a la privacidad y consentimiento informado. Los autores declaran que en este artículo no aparecen datos de pacientes.

\section{Bibliografía}

1. Bustos-Córdova E, Cabrales-Martínez RG, Cerón-Rodríguez $M$, Naranjo-López MY. Epidemiología de lesiones no intencionales en niños: revisión de estadísticas internacionales y nacionales. Bol Med Hosp Infant Mex. 2014;71(2):68-75.

2. Escobar F, Garzón K, Waibel A. Trauma Vascular. En: Benalcázar-Freire JF, Pérez-Guerrero F, editor: Manual de emergencias vasculares. Quito Ecuador: SEACV Capítulo Sierra Norte, 2019; p. 121-40.

3. Yan H, Maximus S, Koopman M, Keeley J, Smith B, de Virgilio C, et al. Vascular trauma operative experience is inadequate in General Surgery programs. Ann Vasc Surg 2016;33:94-7.

4. Inaba K, Aksoy H, Seamon MJ, Marks JA, Duchesne J, Schroll R. et al. Multicenter evaluation of temporary intravascular shunt use in vascular trauma. J Trauma Acute Care Surg. 2016;80(3):359-64.

5. Friend J, Rao S, Sieunarine K, Woodroof P. Vascular trauma in Western Australia: a comparison of wo study periods over 15 years. Anz J Surg. 2016;86:173-8.

6. Williams TK, Fox C, Rasmussen TE. Epidemiology and Natural History of Vascular trauma. En: Cronenwett JL, Johnston KW, ed.: Rutherford's Vascular Surgery. $8^{\text {th }}$ ed. Philadelphia: Saunders; 2014. p. 2350-64.

7. Forrester JD, Weiser TG, Maggio P, Browder T, Tennakoon L, Spain D, et al. Trends in open vascular surgery for trauma: implications for the future of acute care surgery. J Surg Res. 2016;205(1):208-12.

8. Hornez E, Boddaert G, Ngabou UD, Aguir S, Baudoin Y, Mocellin N, et al. Temporary vascular shunt for damage control of extremity vascular injury: A toolbox for trauma surgeons. J Visc Surg. 2015;152(6):363-8.

9. He JC, Clancy K, Schechtman D, Conrad-Schnetz KJ, Claridge JA. Traumatic vascular injuries: who are repairing them and what are the outcomes? Am J Surg. 2016;211(3):619-25.

10. Ramdas MJ, Harnarayan P. A decade of major vascular trauma: lessons learned from gang and civilian warfare. Ann R Coll Surg Engl. 2017;(99)1:70-5.

11. Rodríguez-Lopez E, Fabian-Mijangos W, Casares-Bran T, Lecuona-Huet N, Olivares-Cruz S, Carbajal-Robles V, et al. Trauma vascular civil: tres años de manejo en el Hospital General de México. Rev Mex Angiol. 2017;45(4):154-62.

12. García Núñez LM, Cabello-Pasini R, Decuir-Díaz A, Lever-Rosas CD, Padilla-Solis R, Gómez-García MA, et al. Lesiones vasculares periféricas complejas y shunts intravasculares temporales. El concepto y la institución del "control de daños" van mucho más allá del abdomen. Rev Sanid Milit Mex. 2005;59(4):201-7.

13. Feliciano D, Maryland E. For the patient- Evolution in the management of vascular trauma. J Trauma Acute Care Surg. 2017;83(6):1205-12.

14. Campero-Urcullo A, Córdova-Quintal P, Santillán-Aguayo E. Presentación de Casos: Tratamiento de complicaciones tardías de trauma vascular en el Hospital General de México "Dr. Eduardo Liceaga". Rev Cient Cienc Med. 2014;17(1):47-50.

15. De Olivera Gés A, Vasconcelos R, Brito F, Carvalho M, Vieira S. Vascular trauma in the Amazon- the challenge of great distance. Rev Col Bras Cir. 2015;4(4):244-52.

16. Castañeda-Gaxiola R. The problem of vascular trauma in Mexico. Cir Gen. 2000;22(3):254-6.

17. Friend J, Rao S, Sieunarine K, Woodroof P. Vascular Trauma en Western Australia: a comparison of two study periods over 15 years. Anz J Surg. 2016;86;173-8.

18. Rasmussen TE, TAI NRM. Epidemiology of Vascular Injury. En: Rasmussen TE, TAI NRM, ed. Rich's Vascular Trauma. 3..$^{\text {a }}$ ed. Philadelphia: Elsevier; 2015. p. 13-20.

19. Myers SI, Harward TR, Maher DP, Melissinos EG, Lowry PA. Complex upper extremity vascular trauma in an urban population. $J$ Vasc Surg. 1990;12:305-9.

20. Diamond S, Gaspard D, Katz S. Vascular injuries to the extremities in a suburban trauma center. Am Surg. 2003;69(10):848-51.

21. Espinoza E, Castañeda E. Características clínicas de los traumas vasculares periféricos en pacientes atendidos en un hospital general de Lima, Perú. Rev Med Hered. 2014;25:122-8.

22. Salas DC. Trauma vascular, visión del cirujano vascular. Rev Med Clin Condes. 2011;22(5):686-96.

23. Franz RW, Goodwin RB, Hartman JF, Wright ML. Tratamiento de las lesiones arteriales del miembro superior en un centro metropolitano de traumatología de nivel 1. Ann Vasc Surg. 2009;23(1):8-16. 